\title{
Semi-algebraic geometry of common lines
}

\author{
David Dynerman
}

Correspondence:

dynerman@math.wisc.edu

Department of Mathematics,

University of Wisconsin, 480 Lincoln

Drive, 53706 Madison, WI, USA

\begin{abstract}
Purpose: Cryo-electron microscopy is a technique in structural biology for determining the 3D structure of macromolecules. A key step in this process is detecting common lines of intersection between unknown embedded image planes. We wish to characterize such common lines in terms of the unembedded geometric data detected in experiments.
\end{abstract}

Methods: We use techniques from spherical geometry, real algebraic geometry, and linear algebra.

Results: We show that common lines are the solutions to a system of polynomial equalities and inequalities, i.e., they form a semi-algebraic set. These polynomials are low degree, and we explicitly derive them in this paper.

Conclusions: The polynomials we derive provide the desired intrinsic characterization of common lines. We discuss possible applications of these polynomials to reconstruction algorithms that are robust to the high levels of noise present in cryo-electron images.

Keywords: Cryo-EM; Common lines; Semi-algebraic geometry

\section{Background}

Cryo-electron microscopy (cryo-EM) is a technique used to discover the structure of small molecules, usually proteins in the context of structural biology research [1].

A basic outline of cryo-EM is presented in Figure 1. First, a sample is prepared by freezing many different copies of the molecule in a thin layer of ice. A stream of electrons then passes through the sample and is detected by cameras that produce $N$ noisy 2D cryo-EM images $I_{1}, \ldots, I_{N}$. The primary goal is to reconstruct the 3D structure of the molecule from the 2D images that are acquired. For a more detailed overview, see [2], Section 1.

Problem 1 (Reconstruction problem: structural biology). Given $N$ two-dimensional experimental cryo-EM images $I_{1}, \ldots, I_{N}$, reconstruct a three-dimensional model of the original molecule.

\section{Mathematical model}

We briefly describe the mathematical model for cryo-EM, following [3], Section 0. We work in the three-dimensional space $\mathbb{R}^{3}$ equipped with the usual inner product. The molecule is modeled by a function $\phi: \mathbb{R}^{3} \rightarrow \mathbb{R}$ that represents its electronic density at various spatial locations (Figure 2a). An actual cryo-EM experiment obtains a single 


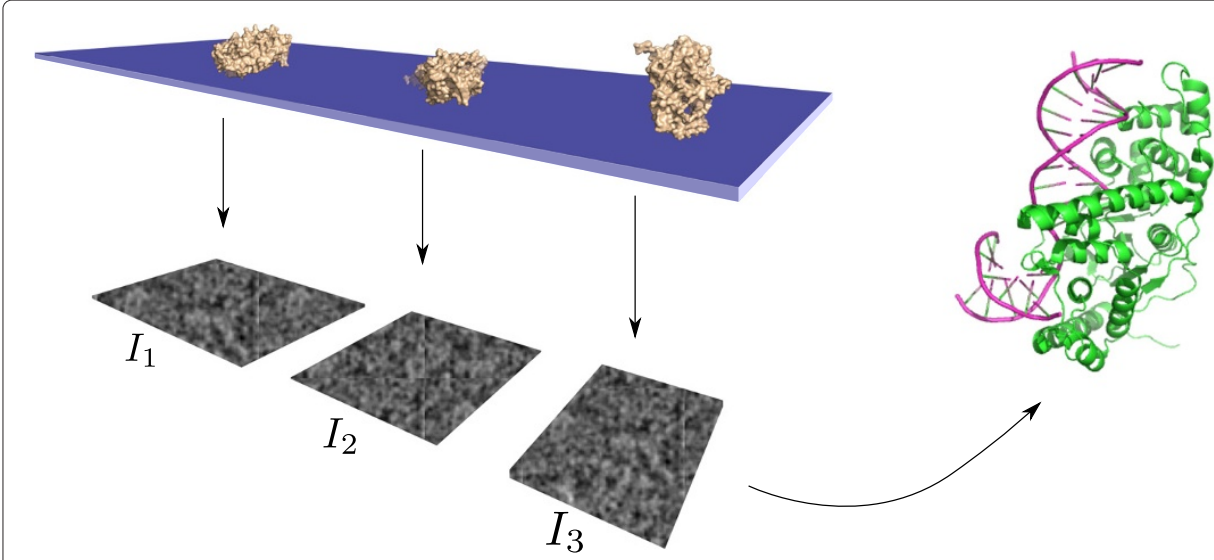

Figure 1 Cryo-EM obtains a 3D structure from noisy 2D images $l_{1}, \ldots, l_{N}$.

image of many copies of the molecule, but we instead assume that each image is a picture of the same molecule from different microscope orientations (Figure 2b). To model a microscope orientation, we use the following concept:

Definition 1. A frame $F$ for $\mathbb{R}^{3}$ is an ordered orthonormal basis $(a, b, c)$ such that the determinant of the matrix $[a b c]$ is +1 or, equivalently, that $c=a \times b$, where $\times$ is the standard cross product on $\mathbb{R}^{3}$.

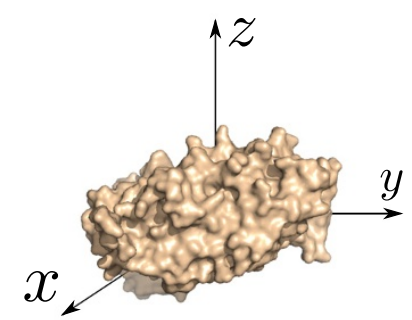

(a) Molecule $\phi: \mathbb{R}^{3} \rightarrow \mathbb{R}$.

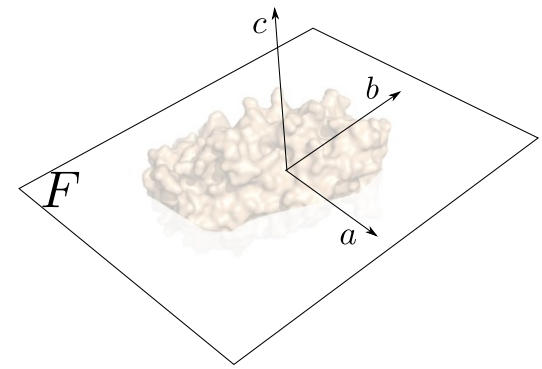

(c) Microscope orientation $F$.

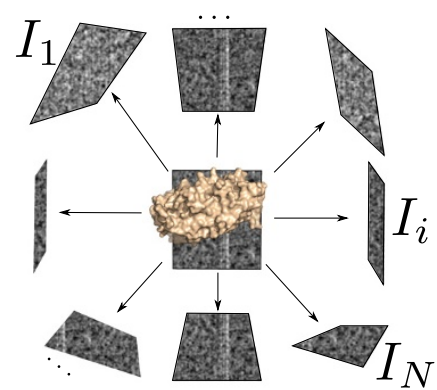

(b) Many images of a single molecule.

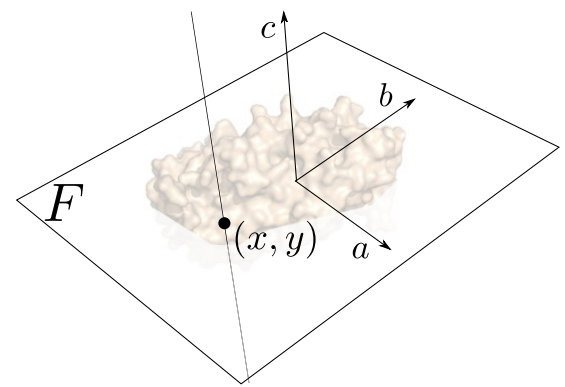

(d) Cryo-EM image from orientation $F$.

Figure 2 Cryo-EM mathematical model. 
Remark 1. A frame $F$ for $\mathbb{R}^{3}$ is uniquely determined by the vectors $(a, b)$. For the rest of the paper, we identify frames $(a, b, c)$ with pairs of orthonormal vectors $(a, b)$.

For us, a microscope orientation is a frame $F=(a, b)$. We think of the span of the vectors $a$ and $b$ as the embedded image plane of this orientation, and the vector $c=a \times b$ as the 'viewing' direction (Figure 2c).

A cryo-EM experiment produces $N$ images which we denote $I_{1}, \ldots, I_{N}$ (see Figure $2 \mathrm{~b}$ ). We will write $F_{i}=\left(a_{i}, b_{i}\right)$ for the microscope orientation of image $I_{i}$. The embedded image plane spanned by $a_{i}, b_{i}$ can be canonically identified with the plane $P_{i}=\mathbb{R}^{2}$. We think of $P_{i}$ as the unembedded image plane of $I_{i}$. We model the image $I_{i}$ as a real valued function on $P_{i}=\mathbb{R}^{2}$. The value of the image $I_{i}$ at the point $(x, y)$ is the integral of $\phi$ along a line perpendicular to the embedded image plane $\operatorname{span}\left\{a_{i}, b_{i}\right\}$ (see Figure $2 \mathrm{~d}$ and Equation 1 ). This is the $X$-ray transform of $\phi$ onto the frame $F_{i}$, given by

$$
\begin{aligned}
I_{i}: P_{i} & =\mathbb{R}^{2} \rightarrow \mathbb{R}, \\
I_{i}(x, y) & =\int_{-\infty}^{\infty} \phi\left(x a_{i}+y b_{i}+z c_{i}\right) d z,
\end{aligned}
$$

where $c_{i}=a_{i} \times b_{i}$. As in [3], to solve this reconstruction problem, we assume that the Xray projections $I_{i}$ and $I_{j}$ of $\phi$ from different microscope orientations $F_{i}$ and $F_{j}$ are different. This is equivalent to requiring the molecule $\phi$ to admit no non-trivial symmetry as a function on $\mathbb{R}^{3}$.

In terms of this mathematical model, the goal of cryo-EM reconstruction (Problem 1) becomes to recover the function $\phi$ from the $N$ X-ray projections $I_{1}, \ldots, I_{N}$. A commonly used approach for this problem is to first recover the $N$ projection orientations $F_{1}, \ldots, F_{N}$ ([3], Section 0.1). Note that the detected image $I_{i}$ is a function on the plane $P_{i}=\mathbb{R}^{2}$, and a cryo-EM experiment does not directly provide information about the microscope orientation $F_{i}$ used to compute $I_{i}$.

Once the original microscope orientations are known, the unembedded image data $I_{1}, \ldots, I_{N}$ can be placed in the original positions from where these X-ray projections were computed. Then the X-ray transform can be inverted to yield an approximation of $\phi$. Thus, although the ultimate goal is to solve Problem 1, we instead discuss solutions to the following problem.

Problem 2 (Reconstruction problem: microscope orientations). Given $N$ X-ray projections $I_{1}, \ldots, I_{N}$ of a molecule $\phi: \mathbb{R}^{3} \rightarrow \mathbb{R}$, computed from the $N$ unknown microscope orientations $F_{1}, \ldots, F_{N}$, recover these orientations up to global rotation.

Remark 2. By 'up to global rotation' we mean that instead of recovering the molecule $\phi$, we instead recover the molecule $\phi$ rotated by an element $R$ in $\mathrm{O}(3)$, the group of $3 \times 3$ orthogonal matrices. The matrix $R$ may be a proper ( $\operatorname{det} R=+1$ ) or improper (det $R=-1$ ) rotation, so we expect chiral ambiguity in the reconstructed molecule.

\section{Common lines and reconstruction}

One approach for solving Problem 2 is to exploit common lines of intersection between the embedded image planes, which we now describe. A cryo-EM experiment produces 
images $I_{i}$ and $I_{j}$ from orientations $F_{i}=\left(a_{i}, b_{i}\right)$ and $F_{j}=\left(a_{j}, b_{j}\right)$. These frames define isometric embeddings $\iota_{i}$ and $\iota_{j}$ (Figure 3) of the unembedded image planes $P_{i}$ and $P_{j}$ into $\mathbb{R}^{3}$, given by

$$
\iota_{i}(x, y)=x a_{i}+y b_{i}, \quad \iota_{j}(x, y)=x a_{j}+y b_{j} .
$$

The images are functions on $P_{i}$ and $P_{j}$, and we know that they were obtained as X-ray projections onto the unknown embedded image planes $\iota_{i}\left(P_{i}\right)$ and $\iota_{j}\left(P_{j}\right)$ (Figure $3 \mathrm{~b}$ ). We assume that each embedded image plane $\iota_{i}\left(P_{i}\right)$ is distinct and, further, that each pair of such planes intersects in a distinct line. Such a configuration of microscope orientations is called generic. The microscope orientations will be generic if they are sampled uniformly from the space of all frames as, for example, assumed in the eigenvector relaxation algorithm developed in [2], Section 3.

The embedded image planes $\iota_{i}\left(P_{i}\right)$ and $\iota_{j}\left(P_{j}\right)$ intersect in a line $L$, see Figure $3 \mathrm{~b}$, and this line corresponds to the unembedded lines $\ell_{i j} \subset P_{i}$ and $\ell_{j i} \subset P_{j}$, see Figure 3a. Since these unembedded lines both came from $L \subset \mathbb{R}^{3}$, we have a natural choice $\psi_{i j}: \ell_{i j} \rightarrow \ell_{j i}$ of one of the two possible isometries between $\ell_{i j}$ and $\ell_{j i}$. Proceeding in this fashion, the $N$ microscope orientations $F_{1}, \ldots, F_{N}$ produce $\left(\begin{array}{c}N \\ 2\end{array}\right)=N(N-1) / 2$ common line pairs $\left\{\left(\ell_{i j}, \ell_{j i}, \psi_{i j}\right)\right\}$. This is the common lines data realized by the frames $F_{1}, \ldots, F_{N}$. It will be useful for us to distinguish such common lines data obtained from frames.

Definition 2. A common line pair for $P_{i}$ and $P_{j}$ is a pair of lines $\ell_{i j} \subset P_{i}$ and $\ell_{j i} \subset P_{j}$, together with a choice of isometry $\psi_{i j}: \ell_{i j} \rightarrow \ell_{j i}$. A collection of common line pairs $\left\{\left(\ell_{i j}, \ell_{j i}, \psi_{i j}\right)\right\}$, for every $P_{i}$ and $P_{j}$, is common lines data for $P_{1}, \ldots, P_{N}$. We say common lines data is valid if it is realized by some generic frames $F_{1}, \ldots, F_{N}$.

Despite the fact that common lines data is the information in the unembedded planes $P_{i}$, it is a fact that, when $N \geq 3$, valid common lines data determines its realizing frames, up to global rotation. Further, algorithms have long been known (e.g., [4], Section 2.1) that recover a set of realizing frames from valid common lines data.

This is relevant to cryo-EM reconstruction, because although the microscope orientations are unknown, it is possible to detect the common lines data the orientations realize

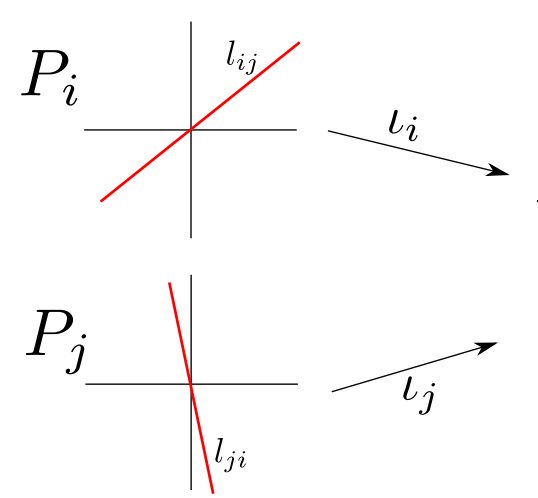

(a)

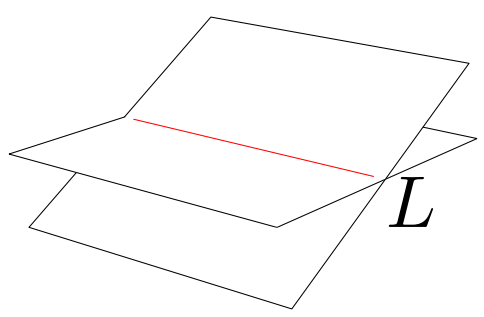

(b)

Figure 3 Common line of $F_{i}$ and $F_{j}$. 
from the images $I_{1}, \ldots, I_{N}$ [5]. Thus, we have the following common lines approach for the cryo-EM reconstruction problem (Problem 2). We first detect the common lines data realized by the unknown microscope orientations. Next, from the valid common lines data, we reconstruct a set of realizing frames. Since valid common lines data determines its realizing frames up to global rotation, the reconstructed frames are related to the original microscope orientations by a global rotation, and so in principle, one has solved the reconstruction problem.

\section{Angular reconstitution}

In this section, we describe the angular reconstitution algorithm, due to van Heel [4], and also independently Vainshtein and Goncharov [6], which recovers a set of realizing frames from valid common lines data.

Our input is valid common lines data $\left\{\left(\ell_{i j}, \ell_{j i}, \psi_{i j}\right)\right\}$ for $P_{1}, \ldots, P_{N}$ (Figure 4$)$. Note that recovering a frame $F_{i}$ is equivalent to recovering the embedding $\iota_{i}$ of $P_{i}$, which will be easier to visualize. Since we are only reconstructing up to global rotation, the first step is to embed $P_{1}$ in an arbitrary position in $\mathbb{R}^{3}$ (Figure $5 \mathrm{a}$ ). Next, we use the isometry $\psi_{12}$ between $\ell_{12}$ and $\ell_{21}$ to dock $P_{2}$ to $\iota_{1}\left(P_{1}\right)$ (Figure $5 \mathrm{~b}$ ). This docking is ambiguous (Figure 5c) since we are free to rotate $\iota_{2}\left(P_{2}\right)$ about its line of intersection with $\iota_{1}\left(P_{1}\right)$. We resolve this ambiguity by docking $P_{3}$ with $\iota_{1}\left(P_{1}\right)$ and matching up $\ell_{23}$ and $\ell_{32}$ in $\iota_{2}\left(P_{2}\right)$ and $\iota_{3}\left(P_{3}\right)$, respectively (Figure $5 \mathrm{~d}$ ). We continue in this fashion, docking each subsequent plane $P_{i}$ with $\iota_{1}\left(P_{1}\right)$ and resolving the rotational ambiguity by comparing against the remaining frames.

\section{Noise and valid common lines data}

We discussed in the 'Common lines and reconstruction' section that valid common lines data determines its realizing frames up to global rotation. Common lines based approaches for cryo-EM reconstruction (Problem 2) assume that we can accurately detect the valid common lines realized by the unknown microscope orientations. Unfortunately, cryo-EM images are very noisy (Figure 6), so we cannot expect to correctly identify common lines data.

Misdetected common lines pose a problem because they lead to inconsistencies when attempting to recover realizing frames. For example, in Figure 5, we resolved the ambiguity of $\iota_{2}\left(P_{2}\right)$ by docking $P_{3}$ to $\iota_{1}\left(P_{1}\right)$ and using the common lines $l_{23}$ and $l_{32}$ (Figure $5 \mathrm{c}$ ). However, we could have equally well resolved the ambiguity of $\iota_{2}\left(P_{2}\right)$ by docking $P_{4}$ and using the common lines $l_{24}$ and $l_{42}$. Thus, if we, for example, incorrectly identify the common lines in $P_{4}$, we will have two contradictory embeddings $\iota_{2}\left(P_{2}\right)$ with no obvious way of determining which is correct. More generally, the angular reconstitution algorithm makes
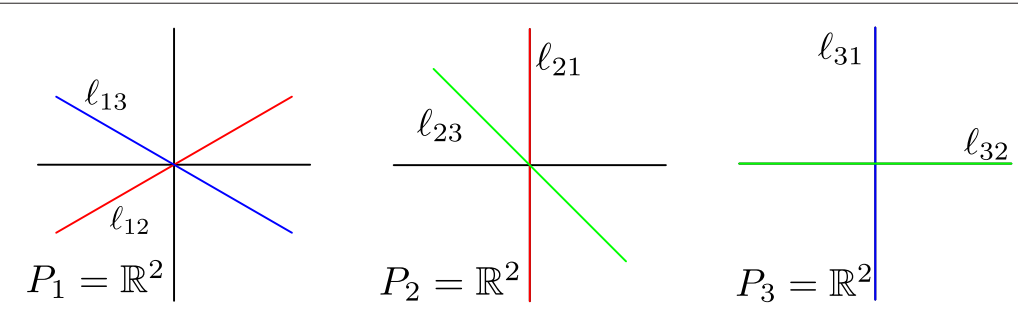

Figure 4 Common lines data for $N=3$ planes. 


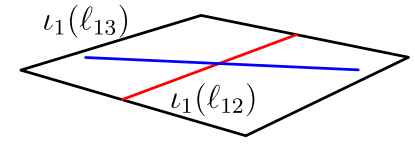

(a)

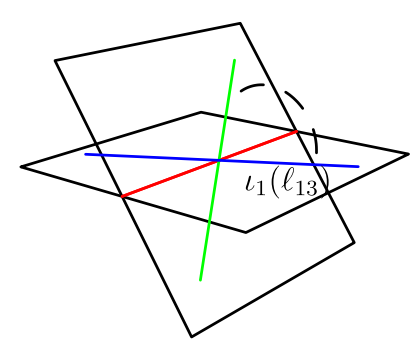

(c)

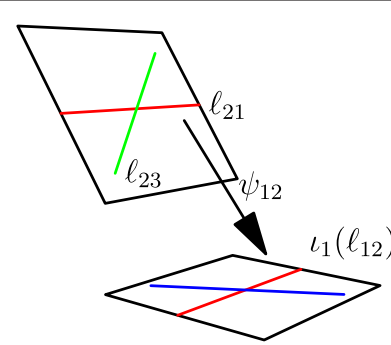

(b)

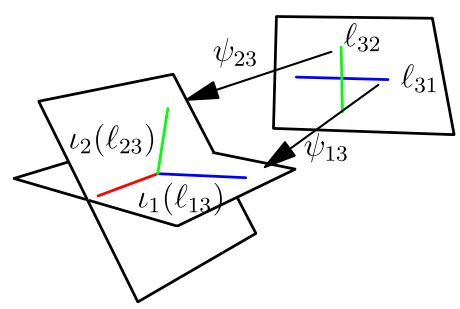

(d)

Figure 5 Angular reconstitution. (a) Place $P_{1}$. (b) Dock $P_{2}$ via $\psi_{12}$. (c) Rotational ambiguity for $\iota_{2}\left(P_{2}\right)$. (d) Resolve ambiguity by docking $P_{3}$ to $\iota_{1}\left(\ell_{13}\right)$.

many choices: for example, which plane to begin reconstruction with and how to resolve docking ambiguities. The final reconstructed frames depend on all these choices. By definition, valid common lines data is precisely the data which has a single consistent (up to global rotation) set of realizing frames. The development of common lines reconstruction algorithms that are robust to this kind of error is an active area of research.

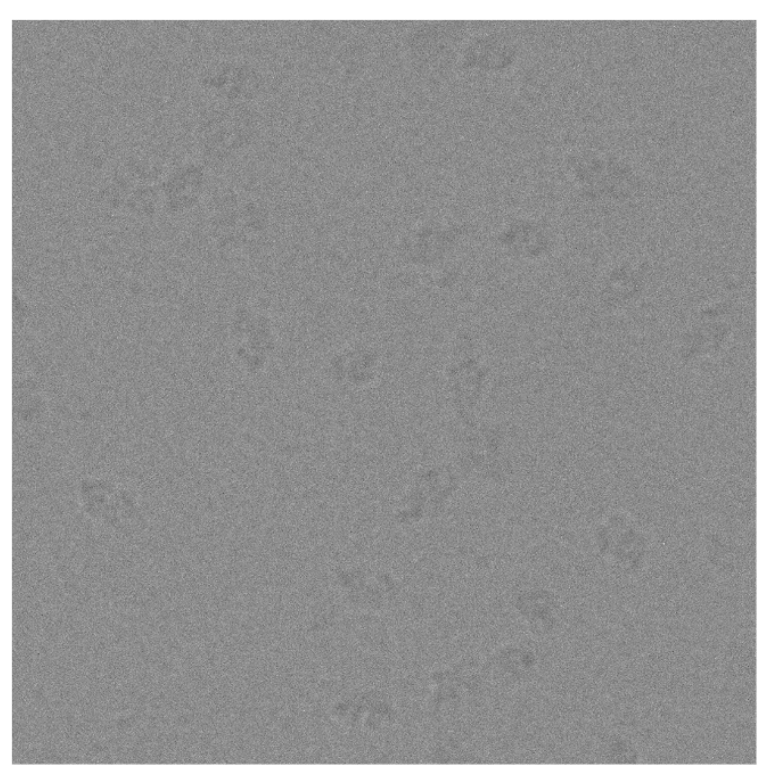

Figure 6 Raw cryo-EM images of $\boldsymbol{\beta}$-galactosidase. Image by Richard Henderson, personal communication. 


\section{Methods}

We wish to understand the set $C_{N}$ of all valid common lines data for $N$ planes $P_{1}, \ldots, P_{N}$. First, we derive necessary and sufficient conditions for common lines data to be valid. These conditions are polynomial equations and inequalities, which means that $C_{N}$ is a semi-algebraic set, and allows us to study $C_{N}$ as a geometric space. In particular, we compute the dimension of $C_{N}$ and show that there is a bijection between $C_{N}$ and the space of generic frames, up to global rotation.

Main Theorem. The set $C_{N}$ of all valid common lines data for $N$ frames is a $3 N-3$ dimensional semi-algebraic subset of the $2\left(\begin{array}{c}N \\ 2\end{array}\right)$ dimensional space of all common lines data and is in bijection with the space of $N$ generic frames modulo $\mathrm{O}(3)$. The defining equations for $C_{N}$ are given by $\left(\begin{array}{c}N \\ 3\end{array}\right)$ polynomial inequalities arising from the spherical triangle inequalities and $6\left(\begin{array}{c}N \\ 4\end{array}\right)$ polynomial equalities arising from the spherical law of cosines.

The meaning of this theorem is as follows: as we discussed in the 'Common lines and reconstruction' section, one way to obtain valid common lines data is from the embedded frames $F_{1}, \ldots, F_{N}$. The theorem provides an intrinsic definition of this valid common lines data, namely, the defining polynomials for $C_{N}$. This is a definition for valid common lines only in terms of the data $\left\{\left(l_{i j}, l_{j i}, \psi_{i j}\right)\right\}$ on unembedded planes $P_{1}, \ldots, P_{N}$ and without reference to any embedded frames $F_{1}, \ldots, F_{N}$.

We briefly describe the idea behind our proofs. Suppose we have valid common lines data

$$
\left\{\left(\ell_{12}, \ell_{21}, \psi_{12}\right),\left(\ell_{13}, \ell_{31}, \psi_{13}\right),\left(\ell_{23}, \ell_{32}, \psi_{23}\right)\right\}
$$

The angles between these unembedded common lines determine a triangle on the unit sphere in $\mathbb{R}^{3}$ (Figure 7), and so the angles $\alpha$ between $\ell_{12}$ and $\ell_{13}, \beta$ between $\ell_{21}$ and $\ell_{23}$, and $\gamma$ between $\ell_{31}$ and $\ell_{32}$ must satisfy the spherical triangle inequalities. These inequalities are analogs of the plane triangle inequality, i.e., necessary and sufficient conditions for a spherical triangle to exist with the specified edge lengths. In other words, a necessary and sufficient condition for common lines data to be valid for $N=3$ is that it satisfies the spherical triangle inequality, a fact already observed both by the cryo-EM ([7], pp. 198-199) and mathematics ([8], Equations 11 and 12) communities.

We prove our results for $N>3$ by similarly appealing to spherical trigonometry. Specifically, given common lines data $\left\{\ell_{i j}, \ell_{j i}, \psi_{i j}\right\}$ for $N$ planes, we require that for each triple
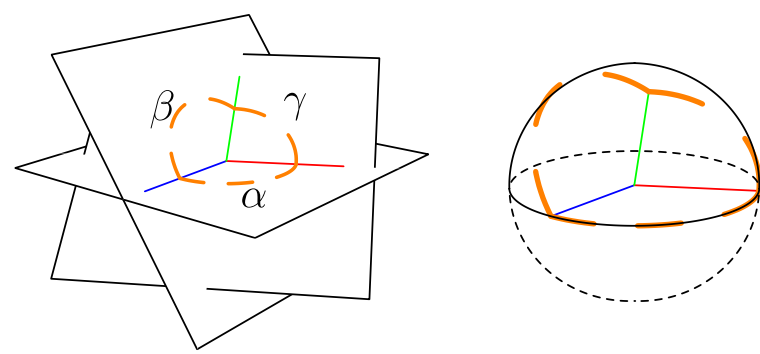

Figure 7 Common lines in $P_{1}, P_{2}$, and $P_{3}$ determine a spherical triangle. 
$1 \leq i<j<k \leq N$, the common lines data $\left(\ell_{i j}, \ell_{j i}, \psi_{i j}\right),\left(\ell_{i k}, \ell_{k i}, \psi_{i k}\right)$, and $\left(\ell_{j k}, \ell_{k j}, \psi_{j k}\right)$ satisfy the spherical triangle inequalities. Now, reducing to the $N=3$ case gives us realizing embeddings $\iota_{i}, \iota_{j}, \iota_{k}$ for each triple $(i, j, k)$ of indices. To reconstruct a collection of $N$ consistent frames, all these triple reconstructions must be compatible. We show that this compatibility condition is a polynomial condition arising from the spherical law of cosines. These defining equations are given by polynomials which are explicitly derived and listed in the 'Defining polynomials' section.

\section{Results and discussion}

We proceed to describe in detail the results in the Main Theorem. We will derive the necessary and sufficient conditions for common lines data for $N \geq 3$ to be valid. These will be explicit polynomial equations and inequalities only in the unembedded information $\left\{\left(l_{i j}, l_{j i}, \psi_{i j}\right)\right\}$ and will provide an intrinsic definition for valid common lines without reference to the frames $F_{1}, \ldots, F_{N}$. We defer all proofs to Appendix 1.

\section{Projective coordinates}

To obtain defining equations for $C_{N}$, it will be convenient for us to work with projective coordinates, which we briefly review. Suppose $V$ is a vector space and $\ell$ is a line in $V$ through the origin. We can represent $\ell$ by choosing any non-zero vector $v \in \ell$. In other words, lines can be identified with equivalence classes of vectors under scaling. We denote the equivalence class of a vector $v$ by $[v]$, and by definition, $[v]=[w]$ if and only $v=\lambda w$, for some $\lambda \neq 0$. The space of all lines through the origin in $V$ is the projective space $\mathbb{P}(V)$. If $V=U \times W$ and $(u, w) \in V$, then we write $[u: w]$ for the corresponding equivalence class in $\mathbb{P}(U \times W)$.

\section{Coordinates for common lines}

Suppose now that $\left(\ell_{i j}, \ell_{j i}, \psi_{i j}\right)$ is a common line pair for $P_{i}$ and $P_{j}$. Choose a non-zero vector $v_{i j}=\left(x_{i j}, y_{i j}\right)$ on the line $\ell_{i j} \subset P_{i}$ and consider the pair $\left(v_{i j}, \psi_{i j}\left(v_{i j}\right)\right) \in P_{i} \times P_{j}$. Note that different choices of a vector along $\ell_{i j}$ will simply scale $\left(v_{i j}, \psi_{i j}\left(v_{i j}\right)\right)$ by a nonzero multiple, so the projective pair $\left[v_{i j}: \psi_{i j}\left(v_{i j}\right)\right]$ in $\mathbb{P}\left(P_{i} \times P_{j}\right)$ is uniquely determined by $\left(\ell_{i j}, \ell_{j i}, \psi_{i j}\right)$.

Conversely, if $\left[v_{i j}: v_{j i}\right] \in \mathbb{P}\left(P_{i} \times P_{j}\right)$ satisfies $\left\|v_{i j}\right\|^{2}=\left\|v_{j i}\right\|^{2}$, then choosing representatives $\left(v_{i j}, v_{j i}\right)$, we obtain a common line pair $\left(\operatorname{span}\left\{v_{i j}\right\}, \operatorname{span}\left\{v_{j i}\right\}, \psi_{i j}\right)$, where $\psi_{i j}$ is the unique isometry that sends $v_{i j} \mapsto v_{j i}$. Note that we obtain the same common line pair regardless of which representing vectors we choose.

Thus, from now on, we identify common line pairs with elements $\left[v_{i j}: v_{j i}\right] \in \mathbb{P}\left(P_{i} \times P_{j}\right)$ satisfying $\left\|v_{i j}\right\|^{2}=\left\|v_{j i}\right\|^{2}$. We also apply this identification to the following common lines data:

Remark 3. We identify common lines data for $P_{1}, \ldots, P_{N}$ with collections

$$
\left(\left[v_{i j}: v_{j i}\right]\right) \in \prod_{1 \leq i<j \leq N} \mathbb{P}\left(P_{i} \times P_{j}\right)=\left(\mathbb{P}^{3}\right)^{\left(\begin{array}{c}
N \\
2
\end{array}\right)}
$$

that satisfy $\left\|v_{i j}\right\|^{2}=\left\|v_{j i}\right\|^{2}$ for all pairs. 
In coordinates, we say that the frames $F_{i}$ and $F_{j}$ realize the common line pair $\left[v_{i j}: v_{j i}\right]$ if the associated embeddings (Equation 2) bring together this common line pair, i.e., for any choice of representative $\left(v_{i j}, v_{j i}\right)$, we have

$$
\iota_{i}\left(v_{i j}\right)=\iota_{j}\left(v_{j i}\right)
$$

By definition, valid common lines data is a collection $\left(\left[v_{i j}: v_{j i}\right]\right)$ of common lines data for which there exist frames $F_{1}, \ldots, F_{N}$ such that for all $1 \leq i<j \leq N$, the frames $F_{i}$ and $F_{j}$ realize $\left[v_{i j}: v_{j i}\right]$.

\section{Necessary and sufficient conditions}

In this section, we derive equations and inequalities that are necessary and sufficient for common lines data $\left(\left(\left[v_{i j}: v_{j i}\right]\right)\right.$ to be valid. We first discuss necessary conditions. Recall from the 'Methods' section that for any triple of indices $i, j, k$ the angles between the common line pairs $\left[v_{i j}: v_{j i}\right],\left[v_{i k}: v_{k i}\right]$, and $\left[v_{j k}: v_{k j}\right]$ determine a spherical triangle (Figure 7), and so these angles must satisfy the spherical triangle inequalities. The spherical triangle inequalities state that a non-degenerate spherical triangle of edge lengths $\alpha, \beta$, and $\gamma$, all in $(0, \pi)$, exists if and only if

$$
\begin{aligned}
\beta+\gamma & >\alpha, \\
\alpha+\gamma & >\beta, \\
\alpha+\beta & >\gamma, \\
\alpha+\beta+\gamma & <2 \pi .
\end{aligned}
$$

Remark 4. Fix common lines data $\left(\left[v_{i j}: v_{j i}\right]\right) \in\left(\mathbb{P}^{3}\right)^{\left(\begin{array}{c}N \\ 2\end{array}\right)}$ and a triple of indices $(i, j, k)$. If we choose representatives $\left(v_{i j}, v_{j i}\right),\left(v_{i k}, v_{k i}\right)$, and $\left(v_{j k}, v_{k j}\right)$, we can write

$$
\begin{aligned}
& \alpha_{i j k}=\cos ^{-1}\left(\frac{v_{i j} \cdot v_{i k}}{\left\|v_{i j}\right\|\left\|v_{i k}\right\|}\right), \beta_{i j k}=\cos ^{-1}\left(\frac{v_{j i} \cdot v_{j k}}{\left\|v_{j i}\right\|\left\|v_{j k}\right\|}\right), \\
& \gamma_{i j k}=\cos ^{-1}\left(\frac{v_{k i} \cdot v_{k j}}{\left\|v_{k i}\right\|\left\|v_{k j}\right\|}\right) .
\end{aligned}
$$

The angles $\alpha_{i j k}, \beta_{i j k}$, and $\gamma_{i j k}$ depend on the representatives we have chosen; however, whether or not the spherical triangle inequalities (Equation 4) are satisfied by $\alpha_{i j k}, \beta_{i j k}$, $\gamma_{i j k}$ is independent of this choice. Thus, we can make the following definition:

Definition 3. Fix common lines data $\left(\left[v_{i j}: v_{j i}\right]\right) \in\left(\mathbb{P}^{3}\right)^{\left(\begin{array}{c}N \\ 2\end{array}\right)}$ and a triple of indices $(i, j, k)$. We say $(i, j, k)$ satisfies the triangle inequalities if, for any choice of representatives $\left(v_{i j}, v_{j i}\right)$, $\left(v_{i k}, v_{k i}\right)$, and $\left(v_{j k}, v_{k j}\right)$, the angles $\alpha_{i j k}, \beta_{i j k}$, and $\gamma_{i j k}$ satisfy Equation 4.

This definition allows us to state our first result.

Proposition 1. Fix common lines data $\left(\left[v_{i j}: v_{j i}\right]\right) \in\left(\mathbb{P}^{3}\right)^{\left(\begin{array}{c}N \\ 2\end{array}\right)}$ and suppose that the triple $(i, j, k)$ satisfies the spherical triangle inequalities. Then, there exist generic frames $F_{i}, F_{j}$, and $F_{k}$ that realize the common line pairs $\left[v_{i j}: v_{j i}\right]$, [ $\left.v_{i k}: v_{k i}\right]$, and $\left[v_{j k}: v_{k j}\right]$. Moreover, if 
$G_{i}, G_{j}$, and $G_{k}$ are another set of frames that realize these same pairs, then there exists an isometry in $\mathrm{O}(3)$ that maps $\left(F_{i}, F_{j}, F_{k}\right) \mapsto\left(G_{i}, G_{j}, G_{k}\right)$.

For a proof of this proposition, see Appendix 1. When $\left(\left[v_{i j}: v_{j i}\right]\right)$ is fixed, and the common lines $\left[v_{i j}: v_{j i}\right]$, $\left[v_{i k}: v_{k i}\right]$ and $\left[v_{j k}: v_{k j}\right]$ are realized by $F_{i}, F_{j}$, and $F_{k}$, we will say that these frames realize the triple $(i, j, k)$.

This proposition is a necessary and sufficient condition for realizing frames to exist for a triple $(i, j, k)$, and so we have recovered a necessary and sufficient conditions for $N=3$. For $N>3$, this proposition states that each triple of indices $(i, j, k)$ must satisfy the spherical triangle inequality, but this condition is no longer sufficient.

Example 1. Consider the common lines data for $P_{1}, P_{2}, P_{3}$, and $P_{4}$ given by

$$
\begin{aligned}
\left(v_{12}, v_{13}, v_{14}\right)=\left(v_{21}, v_{23}, v_{24}\right) & =\left(v_{31}, v_{32}, v_{34}\right)=\left(v_{41}, v_{42}, v_{43}\right) \\
& =\left([1,0]^{T},[\sqrt{2} / 2, \sqrt{2} / 2]^{T},[0,1]^{T}\right) .
\end{aligned}
$$

The angles between these common lines are given by

$$
\begin{array}{ll}
\left(\alpha_{123}, \beta_{123}, \gamma_{123}\right)=\left(\frac{\pi}{4}, \frac{\pi}{4}, \frac{\pi}{4}\right), & \left(\alpha_{124}, \beta_{124}, \gamma_{124}\right)=\left(\frac{\pi}{2}, \frac{\pi}{2}, \frac{\pi}{4}\right), \\
\left(\alpha_{134}, \beta_{134}, \gamma_{134}\right)=\left(\frac{\pi}{4}, \frac{\pi}{2}, \frac{\pi}{2}\right), & \left(\alpha_{234}, \beta_{234}, \gamma_{234}\right)=\left(\frac{\pi}{4}, \frac{\pi}{4}, \frac{\pi}{4}\right) .
\end{array}
$$

Observe that each of these triples satisfies the spherical triangle inequality. However, this data cannot be realized by frames $F_{1}, F_{2}, F_{3}$, and $F_{4}$, and so this common line data is not valid. To see why, suppose such frames existed and, for each pair $i, j$, set $\Lambda_{i j}=$ $\iota_{i}\left(v_{i j}\right)=\iota_{j}\left(v_{j i}\right)$. The points $\Lambda_{12}, \Lambda_{13}$, and $\Lambda_{23}$ determine a spherical triangle with edge lengths $\left(\alpha_{123}, \beta_{123}, \gamma_{123}\right)$ (Figure $\left.8 \mathrm{a}\right)$, and the angle of this spherical triangle at the vertex between edges $\alpha_{123}$ and $\beta_{123}$ is exactly the angle $\theta_{12}$ between the planes $\iota_{1}\left(P_{1}\right)$ and $\iota_{2}\left(P_{2}\right)$. From the spherical law of cosines, we can compute this angle

$$
\cos \theta_{12}=\frac{\cos \gamma_{123}-\cos \alpha_{123} \cos \beta_{123}}{\sin \alpha_{123} \sin \beta_{123}}=\sqrt{2}-1 .
$$

Similarly, the points $\Lambda_{12}, \Lambda_{14}$, and $\Lambda_{24}$ determine a spherical triangle with edge lengths $\left(\alpha_{124}, \beta_{124}, \gamma_{124}\right)$ (Figure 8b), and the angle of this triangle between edges $\alpha_{124}$ and $\beta_{124}$ is again the angle $\theta_{12}$ between the planes $\iota_{1}\left(P_{1}\right)$ and $\iota_{2}\left(P_{2}\right)$. However, in this triangle we have $\cos \theta_{12}=\sqrt{2} / 2$, which is a contradiction.

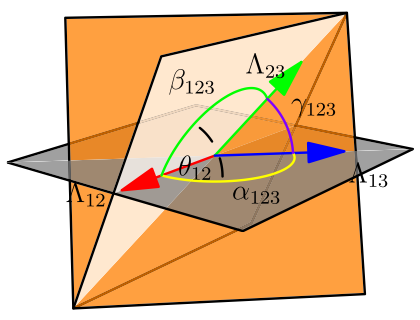

(a) Using $\left[v_{12}: v_{21}\right],\left[v_{13}: v_{31}\right],\left[v_{23}: v_{32}\right]$

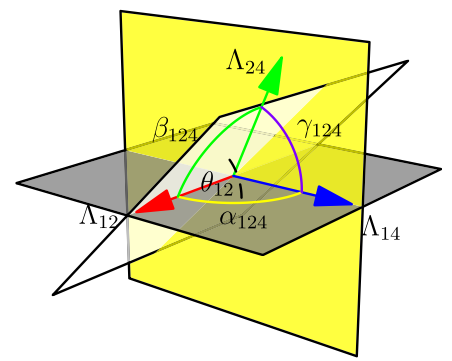

(b) Using $\left[v_{12}: v_{21}\right],\left[v_{14}: v_{41}\right],\left[v_{24}: v_{42}\right]$.

Figure 8 Inconsistent reconstruction from invalid common lines data. 
We now provide an explanation for why the contradiction in Example 1 arose that will lead us to necessary and sufficient conditions for reconstruction when $N>3$. Suppose the frames $F_{1}, \ldots, F_{N}$ realize the common lines data $\left(\left[v_{i j}: v_{j i}\right]\right) \in\left(\mathbb{P}^{3}\right)\left(\begin{array}{c}N \\ 2\end{array}\right)$ and choose unit vector representatives $\left(v_{i j}, v_{j i}\right)$ for all the common line pairs. If we consider the intersection of the embedded planes $\iota_{i}\left(P_{i}\right)$ with the unit sphere in $\mathbb{R}^{3}$, we obtain $N$ great circles. Each pair of these great circles has a distinguished point of intersection $\iota_{i}\left(v_{i j}\right)=\iota_{j}\left(v_{j i}\right)$ which we denote by $\Lambda_{i j}$. Denoted by $T(i, j, k)$, the triangle obtained by taking $\Lambda_{i j}, \Lambda_{i k}$, and $\Lambda_{j k}$ as vertices (Figure 9).

Consider the second triangle $T(i, j, m)$ (Figure 10). The two triangles $T(i, j, k)$ and $T(i, j, m)$ share a vertex, $\Lambda_{i j}$, and the edges of both triangles at this vertex lie in $\iota_{i}\left(P_{i}\right)$ and $\iota_{j}\left(P_{j}\right)$. It follows that the angle $Z$ in $T(i, j, k)$ and $Z^{\prime}$ in $T(i, j, m)$ at this common vertex must be compatible: the angles are either the same (Figure 10a) or supplementary (Figure 10b), depending on the arrangement of the vertices. We can express this requirement in terms of the common lines data by using the spherical law of cosines

$$
\begin{aligned}
& \left(\cos \gamma_{123}-\cos \alpha_{123} \cos \beta_{123}\right) \sin \alpha_{124} \sin \beta_{124}= \\
& \sigma\left(\cos \gamma_{124}-\cos \alpha_{124} \cos \beta_{124}\right) \sin \alpha_{123} \sin \beta_{123},
\end{aligned}
$$

where $\sigma$ determines whether $Z=Z^{\prime}$ or $Z=\pi-Z^{\prime}$. In this light, the contradiction in Example 1 arose because the angles at $\Lambda_{12}$ in $T(1,2,3)$ and $T(1,2,4)$ were not compatible.

Remark 5. Fix common lines data $\left(\left[v_{i j}: v_{j i}\right]\right) \in\left(\mathbb{P}^{3}\right)^{\left(\begin{array}{c}N \\ 2\end{array}\right)}$ and two triples $(i, j, k)$ and $(i, j, m)$ that agree in two indices. If we choose representatives $\left(v_{i j}, v_{j i}\right),\left(v_{i k}, v_{k i}\right),\left(v_{j k}, v_{k j}\right)$, $\left(v_{i m}, v_{m i}\right)$, and $\left(v_{j m}, v_{m j}\right)$ for these common lines, the necessary angle equality (Equation 5$)$ described above is

$$
\begin{aligned}
& L_{i j k, i j m}= \\
& \left(\left(v_{i j} \cdot v_{i j}\right)\left(v_{k i} \cdot v_{k j}\right)-\left(v_{i j} \cdot v_{i k}\right)\left(v_{j i} \cdot v_{j k}\right)\right)\left|\operatorname{det}\left[v_{i j}, v_{i m}\right] \operatorname{det}\left[v_{j i}, v_{j m}\right]\right|- \\
& \quad \sigma\left(\left(v_{i j} \cdot v_{i j}\right)\left(v_{m i} \cdot v_{m j}\right)-\left(v_{i j} \cdot v_{i m}\right)\left(v_{j i} \cdot v_{j m}\right)\right)\left|\operatorname{det}\left[v_{i j}, v_{i k}\right] \operatorname{det}\left[v_{j i}, v_{j k}\right]\right|,
\end{aligned}
$$

where

$$
\sigma=\operatorname{sign}\left(\operatorname{det}\left[v_{i j}, v_{i k}\right] \operatorname{det}\left[v_{i j}, v_{i m}\right] \operatorname{det}\left[v_{j i}, v_{j k}\right] \operatorname{det}\left[v_{j i}, v_{j m}\right]\right) \text {. }
$$

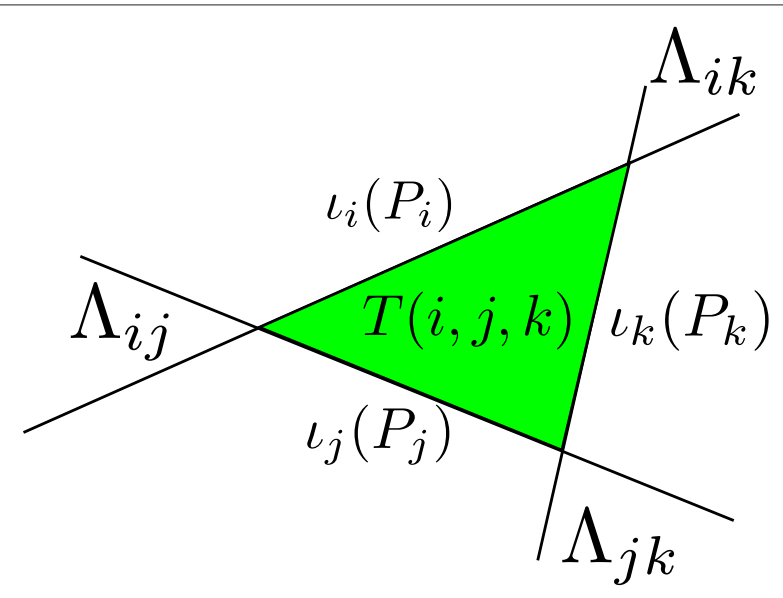

Figure 9 Triangle $T(i, j, k)$ on the surface of the sphere. 


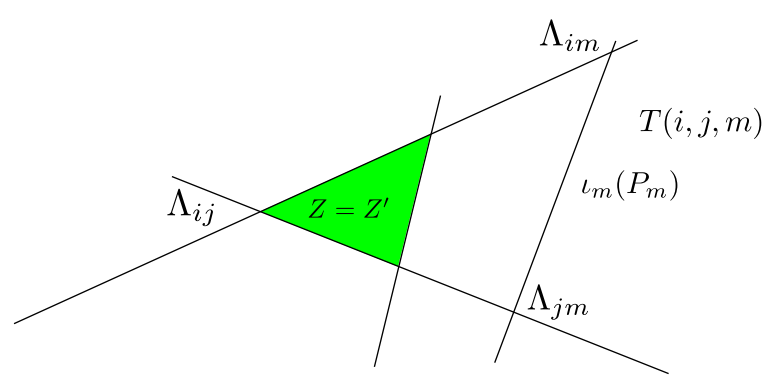

(a) Angle $Z$ in $T(i, j, k)$ equals $Z^{\prime}$ in $T(i, j, m)$.

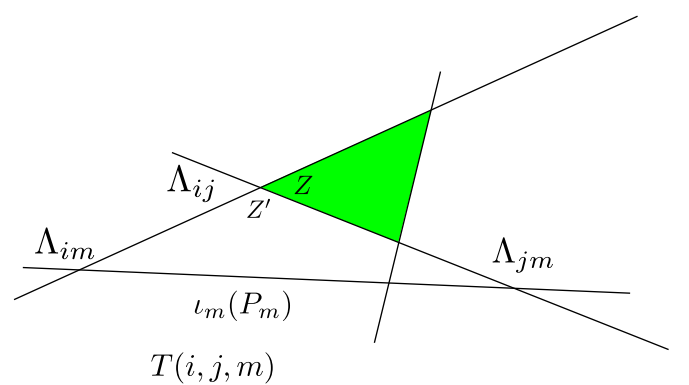

(b) Angle $Z$ in $T(i, j, k)$ is supplementary to $Z^{\prime}$ in $T(i, j, m)$.

Figure $10 T(i, j, k)$, in green, shares edges with $T(i, j, m)$.

Whether or not $L_{i j k, i j m}=0$ is independent of the representatives we choose, we can make the following definition.

Definition 4. Fix common lines data $\left(\left[v_{i j}: v_{j i}\right]\right) \in\left(\mathbb{P}^{3}\right)^{\left(\begin{array}{c}N \\ 2\end{array}\right)}$ and two triples $(i, j, k)$ and $(i, j, m)$ that agree in two indices. We say $(i, j, k)$ and $(i, j, m)$ satisfy the spherical law of cosines compatibility if $L_{i j k, i j m}=0$.

The spherical law of cosines compatibility is necessary for common lines data to be valid, and we will see it is sufficient as well. We first show that if this law of cosines compatibility between $(i, j, k)$ and $(i, j, m)$ is satisfied, then we can glue together realizing frames for these triples in a compatible fashion.

Lemma 1. Fix common lines data $\left(\left[v_{i j}: v_{j i}\right]\right) \in\left(\mathbb{P}^{3}\right)\left(\begin{array}{c}N \\ 2\end{array}\right)$ and suppose that the triples $(i, j, k)$ and $(i, j, m)$ satisfy the spherical law of cosines compatibility. Then, if $F_{i}, F_{j}$, and $F_{k}$ are any realizing frames for $(i, j, k)$, and $G_{i}, G_{j}, G_{m}$ are any realizing frames for $(i, j, m)$, then there exists a unique isometry in $\mathrm{O}(3)$ that sends $F_{i} \mapsto G_{i}$ and $F_{j} \mapsto G_{j}$.

For a proof, see Appendix 1.

We now can show that the law of cosines compatibility is sufficient for reconstruction.

Theorem 1. Fix common lines data $\left(\left[v_{i j}: v_{j i}\right]\right) \in\left(\mathbb{P}^{3}\right)\left(\begin{array}{c}N \\ 2\end{array}\right)$ and suppose that every triple $(i, j, k)$ satisfies the spherical triangle inequality and, further, that every pair of triples 
$(i, j, k)$ and $(i, j, m)$ that agree in two indices satisfies the spherical law of cosines compatibility; then, there exist generic frames $F_{1}, \ldots, F_{N}$, unique up to isometry in $\mathrm{O}(3)$, realizing $\left(\left[v_{i j}: v_{j i}\right]\right)$.

For a proof, see Appendix 1.

\section{Geometry of valid common lines}

We now use the necessary and sufficient conditions derived above to deduce some geometric properties about the set $C_{N}$ of all valid common lines. The main result in this section is that $C_{N}$ is in bijection with the space of generic frames, up to global rotation. In particular, this implies that the dimension of $C_{N}$ is $3 N-3$.

We first explicitly describe how to obtain valid common lines from a set of generic realizing frames $F_{1}, \ldots, F_{N}$, as in the 'Common lines and reconstruction' section. For each pair $i, j$, choose a vector $\Lambda_{i j}$ in the one-dimensional vector space $\iota_{i}\left(P_{i}\right) \cap \iota_{j}\left(P_{j}\right)$. Since $\mathbb{R}^{3}$ has the canonical structure of an inner product space, we have the corresponding orthogonal projections $\iota_{i}^{T}: \mathbb{R}^{3} \rightarrow P_{i}$ and $\iota_{j}^{T}: \mathbb{R}^{3} \rightarrow P_{j}$. Consider the vectors

$$
\left(v_{i j}, v_{j i}\right)=\left(\iota_{i}^{T}\left(\Lambda_{i j}\right), \iota_{j}^{T}\left(\Lambda_{i j}\right)\right) \in P_{i} \times P_{j} .
$$

By construction the pair $\left[v_{i j}: v_{j i}\right]=\left[x_{i j}: y_{i j}: x_{j i}: y_{j i}\right]$ is a common line pair realized by the frames $F_{i}$ and $F_{j}$. In coordinates, we have

$$
x_{i j} a_{i}+y_{i j} b_{i}=\Lambda_{i j}=x_{j i} a_{j}+y_{j i} b_{j} .
$$

Repeating this process for all pairs $1 \leq i<j \leq N$, we obtain valid common lines data $\left(\left[v_{i j}: v_{j i}\right]\right) \in C_{N}$ that is realized by $F_{1}, \ldots, F_{N}$. This algorithmically gives a map $\mathcal{G} \rightarrow C_{N}$, where $\mathcal{G}$ is the subset of $N$ generic frames in $\mathcal{F}^{N}$. It will be useful to express this function via explicit polynomial mappings. We first describe a set of coordinates on the Grassmannian $\operatorname{Gr}(3,2 N)$, whose points are the three-dimensional subspaces of $\mathbb{R}^{2 N}$.

\section{Grassmannian and Plücker coordinates}

If $W \subset \mathbb{R}^{2 N}$ is a three-dimensional subspace of $\mathbb{R}^{2 N}$, and we choose a basis $w_{1}, w_{2}, w_{3} \in$ $\mathbb{R}^{2 N}$ for $W$, we can represent the point in $\operatorname{Gr}(3,2 N)$ corresponding to $W$ by the vector of all $3 \times 3$ minors of the $3 \times 2 N$ matrix

$$
\left[w_{1}, w_{2}, w_{3}\right]^{T} .
$$

These minors are the Plücker coordinates of the subspace $W$. If we choose a different basis for $W$, the vector of $3 \times 3$ minors will only change by a non-zero scalar. Since Plücker coordinates are only defined up to scaling, we interpret the Grassmannian $\operatorname{Gr}(3,2 N)$ as a subvariety of the projective space $\mathbb{P}^{\left(\begin{array}{c}2 N \\ 3\end{array}\right)-1}$.

Given a collection of $N$ frames $F_{1}, \ldots, F_{N}$, we can form the $3 \times 2 N$ matrix

$$
F_{\bullet}=\left[F_{1} \ldots F_{N}\right]=\left[a_{1}, b_{1}, \ldots, a_{N}, b_{N}\right] .
$$

We consider the rational map $\rho: \mathcal{F}^{N} \rightarrow \operatorname{Gr}(3,2 N)$ that takes a collection of frames $F_{1}, \ldots, F_{N}$ to the Plücker coordinates of $F_{0}$. A rational map is a map that is defined almost everywhere in the domain. In this case, $\rho$ is not defined if the rank of $F_{\bullet}$ is $\leq 2$, since, in this case, the rows of $F_{\bullet}$ do not determine a three-dimensional subspace of $\mathbb{R}^{2 N}$. 


\section{Plücker coordinates for common lines}

As described above, given a pair of frames $F_{i}, F_{j}$ for $i<j$, we can compute the associated common line pair $\left[v_{i j}: v_{j i}\right]$ by choosing any vector $\Lambda_{i j}$ in $\iota_{i}\left(P_{i}\right) \cap \iota_{j}\left(P_{j}\right)$. In particular, we can choose $\Lambda_{i j}=\left(a_{i} \times b_{i}\right) \times\left(a_{j} \times b_{j}\right)$, where $\times$ is the standard vector cross product on $\mathbb{R}^{3}$. Then, the following identity from the vector algebra, called the vector quadruple product, expresses $\Lambda_{i j}$ in terms of the frames $F_{i}$ and $F_{j}$

$$
\begin{aligned}
\operatorname{det}\left[a_{j}, b_{j}, a_{i}\right] b_{i}-\operatorname{det}\left[a_{j}, b_{j}, b_{i}\right] a_{i} & =\left(a_{i} \times b_{i}\right) \times\left(a_{j} \times b_{j}\right) \\
& =\operatorname{det}\left[a_{i}, b_{i}, b_{j}\right] a_{j}-\operatorname{det}\left[a_{i}, b_{i}, a_{j}\right] b_{j} .
\end{aligned}
$$

Comparing this with Equation 7, we see that the coordinates of the common line pair $\left[v_{i j}: v_{j i}\right]$ are given by determinants of certain $3 \times 3$ matrices. Explicitly, we have

$$
v_{i j}=\left[\begin{array}{r}
-\operatorname{det}\left[a_{j}, b_{j}, b_{i}\right] \\
\operatorname{det}\left[a_{j}, b_{j}, a_{i}\right]
\end{array}\right], \quad v_{j i}=\left[\begin{array}{r}
\operatorname{det}\left[a_{i}, b_{i}, b_{j}\right] \\
-\operatorname{det}\left[a_{i}, b_{i}, a_{j}\right]
\end{array}\right] .
$$

Observe that these $3 \times 3$ determinants are certain $3 \times 3$ minors of the matrix $F_{\bullet}$. The minors that appear are those that belong to only two frames $F_{i}$ and $F_{j}$, in other words, minors that choose any three of $\left\{a_{i}, b_{i}, a_{j}, b_{j}\right\}$ for columns. The minors not appearing as coordinates of a common line pair are those that choose three columns from three distinct frames

$$
\operatorname{det}\left[\left\{a_{i}, b_{i}\right\},\left\{a_{j}, b_{j}\right\},\left\{a_{k}, b_{k}\right\}\right] .
$$

Thus, the coordinates on the Grassmannian $\operatorname{Gr}(3,2 N)$ are the common line coordinates, together with these 'bad' minors Equation 8. If we consider the projection where we discard the 'bad' minors, we obtain the rational map

$$
\operatorname{Gr}(3,2 N) \rightarrow \prod_{1 \leq i<j \leq N} \mathbb{P}\left(P_{i} \times P_{j}\right)=\left(\mathbb{P}^{3}\right)^{\left(\begin{array}{c}
N \\
2
\end{array}\right)}
$$

Explicitly, for $i<j$, this projection maps

$$
\begin{array}{r}
{\left[\ldots:-\operatorname{det}\left[a_{j}, b_{j}, b_{i}\right]: \operatorname{det}\left[a_{j}, b_{j}, a_{i}\right]: \operatorname{det}\left[a_{i}, b_{i}, b_{j}\right]:-\operatorname{det}\left[a_{i}, b_{i}, a_{j}\right]: \ldots\right]} \\
\end{array}
$$

Note that this rational map is not defined whenever the four $3 \times 3$ minors appearing in the common line pair $\left[v_{i j}: v_{j i}\right]$ simultaneously vanish. This cannot happen with generic frames, so this projection is an actual map when restricted to $\rho(\mathcal{G}) \subset \operatorname{Gr}(3,2 N)$. The image of this map is the set of valid common lines $C_{N}$, and the map is in fact a bijection.

Theorem 2. The restriction $\pi$ of the projection $\operatorname{Gr}(3,2 N) \rightarrow\left(\mathbb{P}^{3}\right)^{\left(\begin{array}{c}N \\ 2\end{array}\right)}$ to $\rho(\mathcal{G}) \subset$ $\operatorname{Gr}(3,2 N)$ is a bijection onto $C_{N}$.

For a proof, see Appendix 1.

As we discussed above, the point $\rho\left(F_{\bullet}\right) \in \operatorname{Gr}(3,2 N)$ only determines the row space of the matrix $F_{\bullet}=\left[F_{1}, \ldots, F_{N}\right]$. A different basis for this row space is given by multiplying

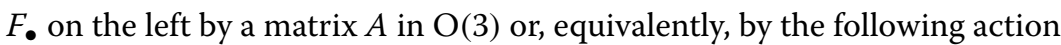

$$
A \cdot\left(F_{1}, \ldots, F_{N}\right)=\left(A F_{1}, \ldots, A F_{N}\right) .
$$


This is the diagonal action of $\mathrm{O}(3)$ on the space of frames $\mathcal{F}^{N}$. We observe that this $\mathrm{O}(3)$ action is the only ambiguity between the space of frames and the Plücker embedding of these frames in $\operatorname{Gr}(3,2 N)$. Since common lines data corresponds to points in $\operatorname{Gr}(3,2 N)$, we have recovered the fact that common lines data only determines its realizing frames up to $\mathrm{O}(3)$.

Corollary 1. The dimension of $C_{N}$ as a semi-algebraic set is $3 N-3$.

For a proof, see Appendix 1.

\section{Conclusions}

The polynomial equations defining $C_{N}$ provide the intrinsic definition for valid common lines we set out to find. We briefly discuss potential applications.

\section{Future work}

Thinking of valid common lines data in geometric terms provides some insight about inconsistencies during reconstruction due to noise. The space of all common lines data has dimension $N(N-1)$, and since valid common lines are in bijection with the space of $N$ frames up to global rotation, we have that the dimension of $C_{N}$ is $3 N-3$. Since $C_{N}$ is a space of small dimension in the ambient space, it follows that the reconstruction inconsistencies described in the 'Noise and valid common lines data' section are guaranteed to occur. In effect, the most basic version of the angular reconstitution algorithm reconstructs the microscope orientations $F_{1}, \ldots, F_{N}$ using only $2 N-3$ out of the $\left(\begin{array}{c}N \\ 2\end{array}\right)$ common line pairs and arbitrarily ignores inconsistencies within these pairs. The set $C_{N}$ is precisely the set of common lines data for which this algorithm will produce the same output regardless of which common line pairs are used, but as described above, we do not expect experimental data to lie in $C_{N}$.

Developing common lines reconstruction algorithms that are robust to noise is an active area of research. We are interested in exploring a geometric approach to noise reduction, which we briefly describe. In principle, noisy experimental data $\left\{\left(l_{i j}^{\prime}, l_{j i}^{\prime}, \psi_{i j}^{\prime}\right)\right\}$ that lies outside of $C_{N}$ 'came from' some noiseless valid common lines data in $C_{N}$. Since the set $C_{N}$ is the set of solutions of a system of polynomials, it is theoretically possible to project noisy common lines to the set of noiseless common lines $C_{N}$ via constrained polynomial optimization. We hope to develop effective projection algorithms along these lines to reduce the impact of noise in reconstruction.

In the 'Results and discussion' section, we obtain defining polynomials for valid common lines data by appealing to spherical geometry. It is also possible to interpret valid common lines in terms of Gram matrices, as in [8] for the case $N=3$. With this interpretation, for $N>3$, one can attempt to find defining polynomials by eliminating certain variables from the defining equations of low rank Gram matrices. The algebraic set corresponding to this elimination is the quotient of $C_{N}$ by the natural action of $\mathrm{SO}(2)^{N}$ in each image plane. We have not yet been able to solve this elimination problem using direct approaches available in the computational algebra software Macaulay2 [9]. We are interested in further studying these related defining polynomials, since they suggest the possibility of applying matrix completion techniques to the denoising projection described above. 


\section{Defining polynomials}

In the 'Necessary and sufficient conditions' section, we derived the defining equations for $C_{N}$ in terms of spherical geometry. For the benefit of the reader, we now explicitly describe these conditions as multi-homogeneous polynomials in the variables $\left(\left[v_{i j}: v_{j i}\right]\right)$.

Suppose $\left(\left[v_{i j}: v_{j i}\right]\right) \in\left(\mathbb{P}^{3}\right)^{\left(\begin{array}{c}N \\ 2\end{array}\right)}$ is fixed, and that $\left\|v_{i j}\right\|^{2}=\left\|v_{j i}\right\|^{2}$ for all $1 \leq i<j \leq$ $N$. The spherical triangle inequalities for the common line pairs $\left[v_{i j}: v_{j i}\right],\left[v_{i k}: v_{k i}\right]$ and $\left[v_{j k}: v_{k j}\right]$ described in Equation 4 are equivalent ([8], Equation 11) to

$$
\begin{gathered}
\left\|v_{i j}\right\|^{2}\left\|v_{i k}\right\|^{2}\left\|v_{j k}\right\|^{2}-\left\|v_{j k}\right\|^{2}\left(v_{i j} \cdot v_{i k}\right)^{2}- \\
\left\|v_{i k}\right\|^{2}\left(v_{j i} \cdot v_{j k}\right)^{2}-\left\|v_{i j}\right\|^{2}\left(v_{k i} \cdot v_{k j}\right)^{2}+ \\
2\left(v_{i j} \cdot v_{i k}\right)\left(v_{j i} \cdot v_{j k}\right)\left(v_{k i} \cdot v_{k j}\right)>0 .
\end{gathered}
$$

To express the spherical law of cosines compatibilities $L_{i j k, i j m}$ (Equation 6), set

$$
\begin{aligned}
a & =\left(\left\|v_{i j}\right\|^{2}\left(v_{k i} \cdot v_{k j}\right)-\left(v_{i j} \cdot v_{i k}\right)\left(v_{j i} \cdot v_{j k}\right)\right), \\
b & =\left(\left\|v_{i j}\right\|^{2}\left(v_{m i} \cdot v_{m j}\right)-\left(v_{i j} \cdot v_{i m}\right)\left(v_{j i} \cdot v_{j m}\right)\right), \\
d_{1} & =\operatorname{det}\left[v_{i j}, v_{i m}\right] \operatorname{det}\left[v_{j i}, v_{j m}\right], \\
d_{2} & =\operatorname{det}\left[v_{i j}, v_{i k}\right] \operatorname{det}\left[v_{j i}, v_{j k}\right] .
\end{aligned}
$$

Then, $L_{i j k, i j m}=0$ if and only if

$$
a^{2} d_{1}^{2}-2 d_{1} d_{2} a b+b^{2} d_{2}^{2}=0 .
$$

Thus, the set $C_{N}$ is defined as a semi-algebraic subset of $\left(\mathbb{P}^{3}\right)\left(\begin{array}{c}N \\ 2\end{array}\right)$ by the following equations and inequalities:

1. The $\left(\begin{array}{c}N \\ 2\end{array}\right)$ equations $\left\|v_{i j}\right\|^{2}=\left\|v_{j i}\right\|^{2}$, see the 'Coordinates for common lines' section.

2. For each of the $\left(\begin{array}{c}N \\ 3\end{array}\right)$ triples $(i, j, k)$, the spherical triangle inequality, see Proposition 1 .

3. For each of the $6\left(\begin{array}{c}N \\ 4\end{array}\right)$ ways to choose two triples of distinct indices $(i, j, k)$ and

$(i, j, m)$ the spherical law of cosines compatibility, see Lemma 1.

\section{Appendix 1 Proofs}

Proof of Proposition 1. Fix representatives $\left(v_{i j}, v_{j i}\right),\left(v_{i k}, v_{k i}\right)$, and $\left(v_{j k}, v_{k j}\right)$. Since the lengths $\alpha_{i j k}, \beta_{i j k}$, and $\gamma_{i j k}$ strictly satisfy the triangle inequalities, there is a non-degenerate spherical triangle with these edge lengths. Denote the vertex of this triangle opposite the edge of length $\alpha_{i j k}$ by $\Lambda_{j k}$, the vertex opposite the edge $\beta_{i j k}$ by $\Lambda_{i k}$ and the vertex opposite the edge $\gamma_{i j k}$ by $\Lambda_{i j}$. Since this triangle is non-degenerate, we know that $\Lambda_{i j}, \Lambda_{i k}$, and $\Lambda_{j k}$ are linearly independent. Thus, we have embeddings $\iota_{i}, \iota_{j}, \iota_{k}$ given by

$$
\begin{aligned}
& \iota_{i}: P_{i} \hookrightarrow \mathbb{R}^{3}, \quad \iota_{j}: P_{j} \hookrightarrow \mathbb{R}^{3}, \quad \iota_{k}: P_{k} \hookrightarrow \mathbb{R}^{3}, \\
& v_{i j} \mapsto \Lambda_{i j}, \quad v_{j i} \mapsto \Lambda_{i j}, \quad v_{k i} \mapsto \Lambda_{i k}, \\
& v_{i k} \mapsto \Lambda_{i k}, \quad v_{j k} \mapsto \Lambda_{j k}, \quad v_{k j} \mapsto \Lambda_{j k} .
\end{aligned}
$$

Observe that these embeddings are isometric by construction, and so $F_{i}=\left(\iota_{i}(x), \iota_{i}(y)\right)$, $F_{j}=\left(\iota_{j}(x), \iota_{j}(y)\right)$, and $F_{k}=\left(\iota_{k}(x), \iota_{k}(y)\right)$ are frames. Since $\Lambda_{i j}, \Lambda_{i k}$, and $\Lambda_{j k}$ are vertices of a non-degenerate spherical triangle, these three frames are in generic position. Moreover, by construction we have

$$
\iota_{i}\left(v_{i j}\right)=\iota_{j}\left(v_{j i}\right), \quad \iota_{i}\left(v_{i k}\right)=\iota_{k}\left(v_{k i}\right), \quad \iota_{j}\left(v_{j k}\right)=\iota_{k}\left(v_{k j}\right),
$$

and so $F_{i}, F_{j}$, and $F_{k}$ realize the required common line pairs. 
Now, suppose $G_{i}, G_{j}$, and $G_{k}$ also realize the common line pairs $\left[v_{i j}: v_{j i}\right],\left[v_{i k}: v_{k i}\right]$ and $\left[v_{j k}: v_{k j}\right]$. Let $\iota_{i}^{G}, \iota_{j}^{G}$, and $\iota_{k}^{G}$ be the embeddings corresponding to these frames and set $\Lambda_{i j}^{G}=\iota_{i}^{G}\left(v_{i j}\right), \Lambda_{i k}^{G}=\iota_{i}^{G}\left(v_{i k}\right)$, and $\Lambda_{j k}^{G}=\iota_{j}^{G}\left(v_{j k}\right)$. Since $(i, j, k)$ strictly satisfies the triangle inequalities, these three vectors are linearly independent and thus define a spherical triangle with edge lengths $\left(\alpha_{i j k}, \beta_{i j k}, \gamma_{i j k}\right)$. This triangle is congruent to the triangle with vertices $\Lambda_{i j}, \Lambda_{i k}$, and $\Lambda_{j k}$ constructed above, and so there exists an element in $\mathrm{O}(3)$ that maps $\Lambda_{i j}^{G} \mapsto \Lambda_{i j}, \Lambda_{i k}^{G} \mapsto \Lambda_{i k}$, and $\Lambda_{j k}^{G} \mapsto \Lambda_{j k}$ and thus maps $\left(G_{i}, G_{j}, G_{k}\right) \mapsto\left(F_{i}, F_{j}, F_{k}\right)$.

Proof of Lemma 1. Fix unit length representatives $\left(v_{i j}, v_{j i}\right),\left(v_{i k}, v_{k i}\right),\left(v_{j k}, v_{k j}\right),\left(v_{i m}, v_{m i}\right)$, and $\left(v_{j m}, v_{m j}\right)$. Let $\iota_{i}^{F}, \iota_{j}^{F}$, and $\iota_{k}^{F}$ be the embeddings corresponding to $F_{i}, F_{j}$, and $F_{k}$ and let $\iota_{i}^{G}, \iota_{j}^{G}$, and $\iota_{m}^{G}$ be the embeddings corresponding to $G_{i}, G_{j}$, and $G_{m}$. Write

$$
\begin{aligned}
\Lambda_{i j}^{F}=\iota_{i}^{F}\left(v_{i j}\right), & \Lambda_{i k}^{F}=\iota_{i}^{F}\left(v_{i k}\right), & \Lambda_{j k}^{F}=\iota_{j}^{F}\left(v_{j k}\right), \\
\Lambda_{i j}^{G}=\iota_{i}^{G}\left(v_{i j}\right), & \Lambda_{i k}^{G}=\iota_{i}^{G}\left(v_{i k}\right), & \Lambda_{j k}^{G}=\iota_{j}^{G}\left(v_{j k}\right) .
\end{aligned}
$$

We wish to show that the map $A: \mathbb{R}^{3} \rightarrow \mathbb{R}^{3}$ defined by

$$
\Lambda_{i j}^{F} \mapsto \Lambda_{i j}^{G}, \quad \Lambda_{i k}^{F} \mapsto \Lambda_{i k}^{G}, \quad \Lambda_{j k}^{F} \mapsto \Lambda_{j k}^{G},
$$

which sends $F_{i} \mapsto G_{i}$ and $F_{j} \mapsto G_{j}$, is an isometry in $\mathrm{O}(3)$. Since $(i, j, k)$ is realized by $F_{i}$, $F_{j}$, and $F_{k}$, it satisfies the spherical triangle inequality, and thus the vectors $\Lambda_{i j}^{F}, \Lambda_{i k}^{F}, \Lambda_{j k}^{F}$ are linearly independent and the map $A$ is uniquely determined. We have that

$$
\Lambda_{i j}^{F} \cdot \Lambda_{i j}^{F}=\Lambda_{i j}^{G} \cdot \Lambda_{i j}^{G}, \quad \Lambda_{i k}^{F} \cdot \Lambda_{i k}^{F}=\Lambda_{i k}^{G} \cdot \Lambda_{i k}^{G}, \quad \Lambda_{j k}^{F} \cdot \Lambda_{j k}^{F}=\Lambda_{j k}^{G} \cdot \Lambda_{j k}^{G},
$$

and further that

$$
\begin{aligned}
& \Lambda_{i j}^{F} \cdot \Lambda_{i k}^{F}=\iota_{i}^{F}\left(v_{i j}\right) \cdot \iota_{i}^{F}\left(v_{i k}\right)=v_{i j} \cdot v_{i k}=\iota_{i}^{G}\left(v_{i j}\right) \cdot \iota_{i}^{G}\left(v_{i k}\right)=\Lambda_{i j}^{G} \cdot \Lambda_{i k}^{G}, \\
& \Lambda_{i j}^{F} \cdot \Lambda_{j k}^{F}=\iota_{j}^{F}\left(v_{j i}\right) \cdot \iota_{j}^{F}\left(v_{j k}\right)=v_{j i} \cdot v_{j k}=\iota_{j}^{G}\left(v_{j i}\right) \cdot \iota_{j}^{G}\left(v_{j k}\right)=\Lambda_{i j}^{G} \cdot \Lambda_{j k}^{G} .
\end{aligned}
$$

It follows that we only need to show that $\Lambda_{i k}^{F} \cdot \Lambda_{j k}^{F}=\Lambda_{i k}^{G} \cdot \Lambda_{j k}^{G}$ to conclude that $A$ is an isometry.

We first discuss the relative orientation of the common line pairs. The product $\operatorname{det}\left[v_{i j}, v_{i k}\right] \operatorname{det}\left[v_{i j}, v_{i m}\right]$ is positive if the shortest rotation from $v_{i j}$ to $v_{i k}$ in $P_{i}$ is in the same direction as the shortest rotation from $v_{i j}$ to $v_{i m}$. In this case, we say $v_{i k}$ and $v_{i m}$ lie on the same side of $v_{i j}$. This product is negative if the shortest rotation from $v_{i j}$ to $v_{i k}$ is in the opposite direction of the shortest rotation of $v_{i j}$ to $v_{i m}$, and in this case, we say $v_{i k}$ and $v_{i m}$ lie on opposite sides of $v_{i j}$. Similarly, the sign of the product det $\left[v_{j i}, v_{j k}\right] \operatorname{det}\left[v_{j i}, v_{j m}\right]$ determines if $v_{j k}$ and $v_{j m}$ lie on the same, or opposite, sides of $v_{j i}$ in $P_{j}$.

Since we consider isometric embeddings of $P_{i}$ and $P_{j}$, we can make the same statements for the embedded versions of these vectors: $\Lambda_{i k}^{G}$ and $\Lambda_{i m}^{G}=\iota_{i}^{G}\left(v_{i m}\right)$ lie on the same side of $\Lambda_{i j}^{G}$ in the plane $\iota_{i}^{G}\left(P_{i}\right)$ if det $\left[v_{i j}, v_{i k}\right] \operatorname{det}\left[v_{i j}, v_{i m}\right]$ is positive, and these vectors lie on opposite sides of $\Lambda_{i j}^{G}$ if this product is negative. We can similarly say whether the vectors $\Lambda_{j k}^{G}$ and $\Lambda_{j m}^{G}=\iota_{j}^{G}\left(v_{j m}\right)$ lie on the same or opposite sides of $\Lambda_{i j}^{G}$ in the plane $\iota_{j}^{G}\left(P_{j}\right)$.

Next, consider the spherical triangle $T$, with vertices $\Lambda_{i j}^{G}, \Lambda_{i k}^{G}$, and $\Lambda_{j k}^{G}$, and the triangle $T^{\prime}$, with vertices $\Lambda_{i j}^{G}, \Lambda_{i m}^{G}$, and $\Lambda_{j m}^{G}$. The triangles $T$ and $T^{\prime}$ share the vertex $\Lambda_{i j}^{G}$, and we write $Z$ for the angle of $T$ at this vertex and $Z^{\prime}$ for the angle of $T^{\prime}$ at this vertex.

Suppose first that $\Lambda_{i k}^{G}$ and $\Lambda_{i m}^{G}$ both lie on the same side of $\Lambda_{i j}^{G}$ in $\iota_{i}^{G}\left(P_{i}\right)$, and $\Lambda_{j k}^{G}$ and $\Lambda_{j m}^{G}$ both lie on the same side of $\Lambda_{i j}^{G}$ in $\iota_{j}^{G}\left(P_{j}\right)$. In this case, the triangles $T$ and $T^{\prime}$ sit inside each other, so $Z$ and $Z^{\prime}$ are the same (cf. Figure 10a). On the other hand, if $\Lambda_{i k}^{G}$ and $\Lambda_{i m}^{G}$ 
lie on opposite sides of $\Lambda_{i j}^{G}$, and $\Lambda_{j k}^{G}$ and $\Lambda_{j m}^{G}$ also lie on opposite sides of $\Lambda_{i j}^{G}$, then the triangle $T^{\prime}$ lies opposite of $T$ across $\Lambda_{i j}^{G}$, so the vertical angles $Z$ and $Z^{\prime}$ are equal. These two cases occur if and only if the quantity,

$$
\sigma=\operatorname{sign}\left(\operatorname{det}\left[v_{i j}, v_{i k}\right] \operatorname{det}\left[v_{i j}, v_{i m}\right] \operatorname{det}\left[v_{j i}, v_{j k}\right] \operatorname{det}\left[v_{j i}, v_{j m}\right]\right) \text {, }
$$

is +1 . Similarly, $\sigma=-1$ if and only if one of the pairs $\Lambda_{i k}^{G}, \Lambda_{i m}^{G}$ or $\Lambda_{j k}^{G}, \Lambda_{j m}^{G}$ lies on the same side of $\Lambda_{i j}^{G}$, while the other pair lies on opposite sides of $\Lambda_{i j}^{G}$. In this case the triangles $T$ and $T^{\prime}$ sit side by side, so the angles $Z$ and $Z^{\prime}$ are supplementary (cf. Figure 10b).

It follows that $\cos Z=\sigma \cos Z^{\prime}$, and so applying the spherical law of cosines in $T$ yields

$$
\frac{\Lambda_{i k}^{G} \cdot \Lambda_{j k}^{G}-\left(v_{i j} \cdot v_{i k}\right)\left(v_{j i} \cdot v_{j k}\right)}{\left|\operatorname{det}\left[v_{i j}, v_{i k}\right] \operatorname{det}\left[v_{j i}, v_{j k}\right]\right|}=\sigma \cos Z^{\prime}
$$

On the other hand, the law of cosines in $T^{\prime}$ gives

$$
\cos Z^{\prime}=\frac{v_{m i} \cdot v_{m j}-\left(v_{i j} \cdot v_{i m}\right)\left(v_{j i} \cdot v_{j m}\right)}{\left|\operatorname{det}\left[v_{i j}, v_{i m}\right] \operatorname{det}\left[v_{j i}, v_{j m}\right]\right|},
$$

and finally, since $L_{i j k, i j m}=0$ we have

$$
\sigma \cos Z^{\prime}=\frac{v_{k i} \cdot v_{k j}-\left(v_{i j} \cdot v_{i k}\right)\left(v_{j i} \cdot v_{j k}\right)}{\left|\operatorname{det}\left[v_{i j}, v_{i k}\right] \operatorname{det}\left[v_{j i}, v_{j k}\right]\right|} .
$$

Thus, we have that $\Lambda_{i k}^{G} \cdot \Lambda_{j k}^{G}=v_{k i} \cdot v_{k i}=\Lambda_{i k}^{F} \cdot \Lambda_{j k}^{F}$, and so $A$ is an isometry, as desired.

Proof of Theorem 2. By Proposition 1, we first obtain realizing frames $F_{1}, F_{2}$, and $F_{3}$ for the triple $(1,2,3)$. For all remaining indices $i$, we construct realizing frames $G_{1}, G_{2}$, and $G_{i}$ from the triple $(1,2, i)$. By Lemma 1 , there exists a unique map $A_{i} \in \mathrm{O}(3)$ that maps $F_{1} \mapsto G_{1}$ and $F_{2} \mapsto G_{2}$. If det $A_{i}=-1$, we can replace the realizing frames $G_{1}, G_{2}$, and $G_{i}$ by $L\left(G_{1}\right), L\left(G_{2}\right)$, and $L\left(G_{i}\right)$, respectively, where $L$ is an arbitrary isometry in $\mathrm{O}(3)$ with det $L=-1$, and replace $A_{i}$ by $L \circ A_{i}$. It follows that we can assume $A_{i}$ has det $=+1$. We set $F_{i}=A_{i}^{-1} G_{i}$.

Now we need to check that the $F_{i}$ are realizing frames. We will write $\iota_{i}^{F}, \iota_{j}^{F}$, and $\iota_{k}^{F}$ for the embeddings determined by $F_{i}, F_{j}$, and $F_{k}$ and similarly for other sets of reconstructed frames. Thus, we need to verify that $\iota_{i}^{F}\left(v_{i j}\right)=\iota_{j}^{F}\left(v_{j i}\right)$ for all pairs $i, j$. To this end, suppose that $F_{i}=A_{i}^{-1} G_{i}$ was reconstructed from $G_{1}, G_{2}$, and $G_{i}$ and $F_{j}=A_{j}^{-1} D_{j}$ was reconstructed from $D_{1}, D_{2}$, and $D_{j}$. The triple $(1, i, j)$ also strictly satisfies the triangle inequality, so we have generic realizing frames $H_{1}, H_{i}$, and $H_{j}$. By Lemma 1 , we have isometries $B_{i}:\left(G_{1}, G_{i}\right) \mapsto\left(H_{1}, H_{i}\right)$ and $B_{j}:\left(D_{1}, D_{j}\right) \mapsto\left(H_{1}, H_{j}\right)$. These maps and frames fit into the following diagram:

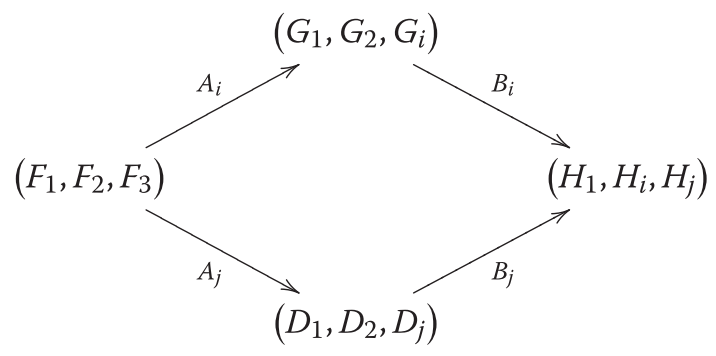


First, note that det $B_{i}= \pm 1$ and det $B_{j}= \pm 1$, and in fact, we claim that det $B_{i}=\operatorname{det} B_{j}$. To see why, write $\Lambda_{12}^{G}=\iota_{1}^{G}\left(v_{12}\right)=\iota_{2}^{G}\left(v_{21}\right)$ and similarly for all other common line pairs. Then, we have

$$
\begin{aligned}
& \left(\operatorname{det} B_{i}\right) \operatorname{sign}\left(\operatorname{det}\left[\Lambda_{12}^{G}, \Lambda_{1 i}^{G}, \Lambda_{2 i}^{G}\right]\right)=\operatorname{sign}\left(\left[\Lambda_{12}^{H}, \Lambda_{1 i}^{H}, \Lambda_{2 i}^{H}\right]\right), \\
& \left(\operatorname{det} B_{j}\right) \operatorname{sign}\left(\operatorname{det}\left[\Lambda_{12}^{D}, \Lambda_{1 j}^{D}, \Lambda_{2 j}^{D}\right]\right)=\operatorname{sign}\left(\left[\Lambda_{12}^{H}, \Lambda_{1 j}^{H}, \Lambda_{2 j}^{H}\right]\right) .
\end{aligned}
$$

Further, if $\sigma=\operatorname{sign}\left(\operatorname{det}\left[v_{12}, v_{1 i}\right] \operatorname{det}\left[v_{12}, v_{1 j}\right] \operatorname{det}\left[v_{21}, v_{2 i}\right] \operatorname{det}\left[v_{21}, v_{2 j}\right]\right)$, we have

$$
\begin{aligned}
& \operatorname{sign}\left(\operatorname{det}\left[\Lambda_{12}^{H}, \Lambda_{1 i}^{H}, \Lambda_{2 i}^{H}\right]\right)=\sigma \operatorname{sign}\left(\operatorname{det}\left[\Lambda_{12}^{H}, \Lambda_{1 j}^{H}, \Lambda_{2 j}^{H}\right]\right), \\
& \operatorname{sign}\left(\operatorname{det}\left[\Lambda_{12}^{G}, \Lambda_{1 i}^{G}, \Lambda_{2 i}^{G}\right]\right)=\sigma \operatorname{sign}\left(\operatorname{det}\left[\Lambda_{12}^{G}, \Lambda_{1 j}^{G}, \Lambda_{2 j}^{G}\right]\right), \\
& \operatorname{sign}\left(\operatorname{det}\left[\Lambda_{12}^{D}, \Lambda_{1 i}^{D}, \Lambda_{2 i}^{D}\right]\right)=\sigma \operatorname{sign}\left(\operatorname{det}\left[\Lambda_{12}^{D}, \Lambda_{1 j}^{D}, \Lambda_{2 j}^{D}\right]\right) .
\end{aligned}
$$

On the other hand, $\operatorname{det}\left(A_{i} \circ A_{j}^{-1}\right)=1$, so

$$
\operatorname{sign}\left(\operatorname{det}\left[\Lambda_{12}^{G}, \Lambda_{1 i}^{G}, \Lambda_{2 i}^{G}\right]\right)=\sigma \operatorname{sign}\left(\operatorname{det}\left[\Lambda_{12}^{D}, \Lambda_{1 j}^{D}, \Lambda_{2 j}^{D}\right]\right)
$$

and thus det $B_{i}=\operatorname{det} B_{j}$. Note that the diagram above commutes, since both the top path and bottom path are morphisms in $\mathrm{O}(3)$ of the same determinant that send $F_{1} \mapsto H_{1}$. Then, since $H_{1}, H_{i}$, and $H_{j}$ realize the common line pair $\left(v_{i j}, v_{j i}\right)$, we have

$$
\begin{aligned}
\iota_{i}^{F}\left(v_{i j}\right)=A_{i}^{-1} \iota_{i}^{G}\left(v_{i j}\right) & =\left(A_{i}^{-1} \circ B_{i}^{-1}\right) \iota_{i}^{H}\left(v_{i j}\right) \\
& =\left(A_{i}^{-1} \circ B_{i}^{-1}\right) \iota_{j}^{H}\left(v_{j i}\right) \\
& =\left(A_{j}^{-1} \circ B_{j}^{-1}\right) \iota_{j}^{H}\left(v_{j i}\right)=A_{j}^{-1} \iota_{j}^{D}\left(v_{j i}\right)=\iota_{j}^{F}\left(v_{j i}\right)
\end{aligned}
$$

and thus $F_{1}, \ldots, F_{N}$ realize the common lines data $\left(\left[v_{i j}: v_{j i}\right]\right)$.

Finally, suppose that $F_{1}^{\prime}, \ldots, F_{N}^{\prime}$ is another collection of realizing frames for $\left(\left[v_{i j}: v_{j i}\right]\right)$. Fix a triple $(i, j, k)$ and observe that since both $F_{i}, F_{j}, F_{k}$ and $F_{i}^{\prime}, F_{j}^{\prime}, F_{k}^{\prime}$ are realizing frame for $(i, j, k)$ by Proposition 1 , there is an isometry $R_{i j k}$ that sends $\left(F_{i}^{\prime}, F_{j}^{\prime}, F_{k}^{\prime}\right) \mapsto\left(F_{i}, F_{j}, F_{k}\right)$. Note that for any $i, j, k, m$, the two isometries $R_{i j k}$ and $R_{i j m}$ are equal since they agree on $F_{i}^{\prime}$ and $F_{j}^{\prime}$. This implies that $R_{i j k}\left(F_{m}^{\prime}\right)=F_{m}$ for all $m$, and thus there is a single isometry $\left(F_{1}^{\prime}, \ldots, F_{N}^{\prime}\right) \mapsto\left(F_{1}, \ldots, F_{N}\right)$.

Proof of Theorem 2. First, observe that the minors corresponding to a common line pair $\left[v_{i j}: v_{j i}\right]$ are non-zero for points in $\rho(\mathcal{G})$, since otherwise $F_{i}$ and $F_{j}$ would define the same plane. It follows that the rational projection $\operatorname{Gr}(3,2 N) \rightarrow\left(\mathbb{P}^{3}\right)\left(\begin{array}{c}N \\ 2\end{array}\right)$ is defined everywhere on $\rho(\mathcal{G})$.

By definition, any valid common lines data $\left(\left[v_{i j}: v_{j i}\right]\right) \in C_{N}$ has some realizing frames $F_{1}, \ldots, F_{N}$, and so is the image of $\pi\left(\rho\left(F_{\bullet}\right)\right)$ and thus $\pi(\rho(\mathcal{G}))=C_{N}$. It only remains to verify that this projection is injective. This follows from Theorem 1. If $\pi\left(\rho\left(F_{\bullet}\right)\right)=$ $\pi\left(\rho\left(G_{\bullet}\right)\right)$, then we know that the realizing frames $F_{\bullet}$ and $G_{\bullet}$ are related by an isometry in $\mathrm{O}(3)$. But then the rows of the matrices $F_{\bullet}$ and $G_{\bullet}$ define the same linear subspace, and so $\rho\left(F_{\bullet}\right)=\rho\left(G_{\bullet}\right)$. 
Proof of Corollary 1. We will compute dimensions with respect to a dense subset of $\mathcal{G}$ and a dense subset of $\rho(\mathcal{G}) \times \mathrm{O}(3)$. Let $V \subset \mathcal{G}$ be the complement of the semi-algebraically homeomorphic to an open subset of $\operatorname{SO}(3)^{N}$ we have $\operatorname{dim} V=\operatorname{dim} \mathcal{G}=3 N$, and thus $\operatorname{dim} \rho(V)=\operatorname{dim} \rho(\mathcal{G})=3 N-3$. By Theorem 2, we have a semi-algebraic bijection between $\rho(\mathcal{G})$ and $C_{N}$, so we conclude that $\operatorname{dim} C_{N}=3 N-3$.

Competing interests

The author declares that he has no competing interests.

\section{Acknowledgments}

The author is greatly thankful to Shamgar Gurevich, for initially suggesting the cryo-EM problem and for his continued support, as well as to Bernd Sturmfels, who suggested studying defining equations in cryo-EM, provided helpful guidance, and invited the author to the Mathematical Sciences Research Institute (MSRI) in Berkeley, California. Much of this work took place at MSRI during the spring of 2013, and the author greatly appreciates helpful technical discussions with Luke Oeding, Kristian Ranestad, Yoel Shkolnisky, Amit Singer, and Frank Sottile. The author's visit to MSRI was supported by the National Science Foundation under grants DMS-0838210 and DMS-0932078.

Received: 11 April 2014 Accepted: 8 October 2014

Published online: 02 December 2014

\section{References}

1. Wang, L, Sigworth, FJ: Cryo-EM and single particles. Physiology. 21(1), 13-18 (2006)

2. Singer, A, Shkolnisky, Y: Three-dimensional structure determination from common lines in cryo-EM by eigenvectors and semidefinite programming. SIAM J. Imag. Sci. 4(2), 543-572 (2011)

3. Hadani, R, Singer, A: Representation theoretic patterns in three dimensional Cryo-Electron Microscopy I: the intrinsic reconstitution algorithm. Ann. Math. 174(2), 1219-1241 (2011). doi:10.4007/annals.2011.174.2.11. Accessed 14 January 2013

4. Van Heel, M: Angular reconstitution: a posteriori assignment of projection directions for 3 reconstruction. Ultramicroscopy. 21(2), 111-123 (1987)

5. De Rosier, D, Klug, A: Reconstruction of three dimensional structures from electron micrographs. Nature. 217(5124), 130-134 (1968)

6. Vainshtein, B, Goncharov, A: Determination of the spatial orientation of arbitrarily arranged identical particles of unknown structure from their projections. In: Soviet Physics Doklady, vol. 31, p. 278. American Institute of Physics, New York (1986)

7. Van Heel, M, Orlova, E, Harauz, G, Stark, H, Dube, P, Zemlin, F, Schatz, M: Angular reconstitution in three-dimensional electron microscopy: historical and theoretical aspects. Scanning Microsc. 11, 195-210 (1997)

8. Singer, A, Coifman, RR, Sigworth, FJ, Chester, DW, Shkolnisky, Y: Detecting consistent common lines in cryo-EM by voting. J. Struct. Biol. 169(3), 312-322 (2010). doi:10.1016/j.jsb.2009.11.003. Accessed 25 February 2014

9. Grayson, DR, Stillman, ME: Macaulay2, a software system for research in algebraic geometry. Available at http://www. math.uiuc.edu/Macaulay2/

\section{Submit your manuscript to a SpringerOpen ${ }^{\circ}$ journal and benefit from:}

- Convenient online submission

- Rigorous peer review

- Immediate publication on acceptance

- Open access: articles freely available online

- High visibility within the field

- Retaining the copyright to your article

Submit your next manuscript at $>$ springeropen.com 